\title{
(GIGA)bYte
}

DATA RELEASE

\section{Bicolor angelfish (Centropyge bicolor) provides the first chromosome-level genome of the Pomacanthidae family}

\author{
Chunhua $\mathrm{Li}^{1, \dagger}$, Xianwei Yang ${ }^{1,3, \dagger}$, Libin Shao ${ }^{1, \dagger}$, Rui Zhang ${ }^{1}$, Qun Liu ${ }^{1}$, \\ Mengqi Zhang ${ }^{1}$, Shanshan Liu ${ }^{1}$, Shanshan Pan ${ }^{1}$, Weizhen Xue ${ }^{1}$, \\ Congyan Wang ${ }^{1}$, Chunyan Mao $^{1}$, He Zhang ${ }^{1,2, *}$ and Guangyi Fan ${ }^{1, *}$ \\ 1 BGI-Qingdao, BGI-Shenzhen, Qingdao 26655-5, China \\ 2 Department of Biology, Hong Kong Baptist University, Hong Kong, China \\ 3 College of Life Sciences, University of Chinese Academy of Sciences, Beijing 100049, China
}

\section{ABSTRACT}

The Bicolor Angelfish, Centropyge bicolor, is a tropical coral reef fish. It is named for its striking two-color body. However, a lack of high-quality genomic data means little is known about the genome of this species. Here, we present a chromosome-level $C$. bicolor genome constructed using Hi-C data. The assembled genome is $650 \mathrm{Mbp}$ in size, with a scaffold N50 value of $4.4 \mathrm{Mbp}$, and a contig N50 value of $114 \mathrm{Kbp}$. Protein-coding genes numbering 21,774 were annotated. Our analysis will help others to choose the most appropriate de novo genome sequencing strategy based on resources and target applications. To the best of our knowledge, this is the first chromosome-level genome for the Pomacanthidae family, which might contribute to further studies exploring coral reef fish evolution, diversity and conservation.

Subjects Genetics and Genomics, Evolutionary Biology, Marine Biology

Submitted: 14 May 2021

Accepted: $\quad 26$ October 2021

Published: $\quad 09$ November 2021

Corresponding authors. E-mail: fanguangyi@genomics.cn; zhanghe@genomics.cn

$\dagger$ Contributed equally.

Published by GigaScience Press.

Preprint submitted at https: //doi.org/10.1101/2021.10.24.465606

This is an Open Access article distributed under the terms of the Creative Commons Attribution License (http://creativecommons.org/ licenses/by/4.0/), which permits unrestricted reuse, distribution, and reproduction in any medium, provided the original work is properly cited.

Gigabyte, 2021, 1-13

\section{DATA DESCRIPTION}

\section{Background}

Centropyge bicolor (NCBI:txid109723; FishbaseID: 5454;

urn:lsid:marinespecies.org:taxname:211780) (Figure 1), also known as the Bicolor, Two-Colored, or Pacific Rock Beauty Angelfish, is a showy coral reef fish commonly distributed in the Indo-Pacific ocean (from East Africa to the Samoan and Phoenix Islands, north to southern Japan, south to New Caledonia; throughout Micronesia). As a member of the Pomacanthidae family, it is similar to those of the Chaetodontidae (Butterflyfishes) but is distinguished by the presence of strong preopercle spines. $C$. bicolor has clear boundaries between its body colors, so might be a good model in which to study body color development in coral fish [1].

\section{Context}

Although the availability of genetic, and especially genomic resources, remains limited for the Pomacanthidae family, we assembled the first $C$. bicolor reference genome. This will provide valuable information for genetic studies of this coral reef fish, and will contribute to studies in body color diversity. With the whole genome sequence of $C$. bicolor, it might be 


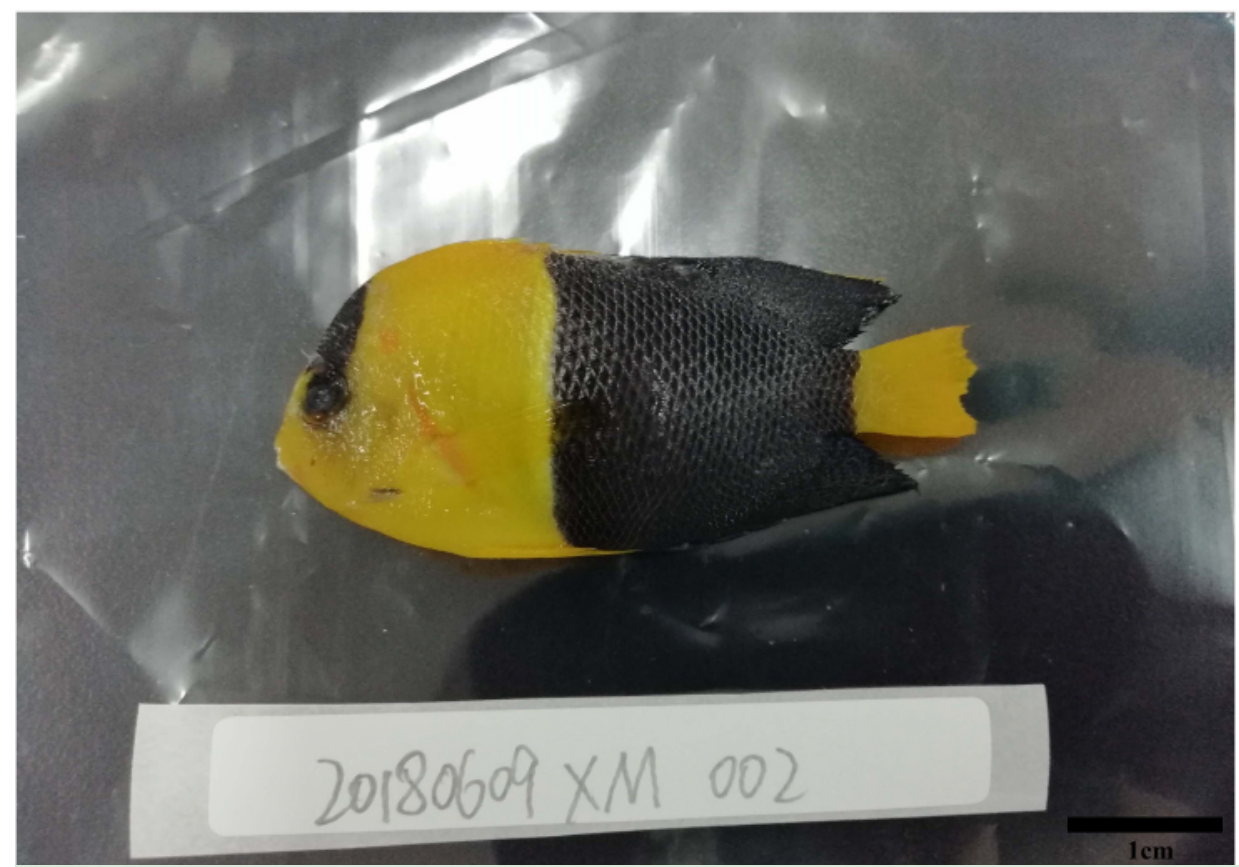

Figure 1. Photograph of Centropyge bicolor.

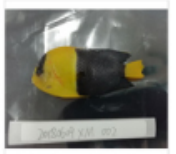

Nov 21, 2020

sookmark

P Copy / Fork

Protocols for "Bicolor Angelfish (Centropyge bicolor) genome provided first chromosome-level reference of Pomacanthidae family and clues for bi-color body formation"

Chunhua Li ${ }^{1}$, Xianwei Yang ${ }^{1}$, Libin Shao ${ }^{1}$, Rui Zhang ${ }^{1}$, QunLiu' ${ }^{1}$, Mengqi Zhang ${ }^{1}$, Shanshan Liu ${ }^{1}$, Shanshan Pan ${ }^{1}$ Weizhen Xue ${ }^{1}$, Congyan Wang ${ }^{1}$, Chunyan Mao' ${ }^{1}$, He Zhang ${ }^{2}$, Guangyi Fan ${ }^{1}$

'BGI-Qingdao, BGI-Shenzhen, Qingdao 266555, China;

${ }^{2}$ BGI-Qingdao, BGI-Shenzhen, Qingdao 266555, China,Department of Biology, Hong Kong Baptist University, Hong Kong, China
1 Works for me
\& Share
dx.doi.org/10.17504/protocols.io.bpxhmpj6
$\therefore$ lichunhua 8

Figure 2. Protocols for BGISEQ-500, stLFR and Hi-C library preparation and construction, and genome assembly, for the Bicolor Angelfish, Centropyge bicolor [2]. https://www.protocols.io/widgets/doi?uri=dx.doi.org/10.17504/ protocols.io.bpxhmpj6

possible to explore the genetic mechanisms of body color development in coral reef fish by comparative genomic methods.

\section{METHODS AND RESULTS}

A protocols collection for BGISEQ-500, stLFR and Hi-C library construction is available in protocols.io (Figure 2) [2].

\section{Sample collection and genome sequencing}

A C. bicolor individual was collected from the market in Xiamen, Fujian Province, China. DNA was extracted from fresh muscle tissue according to a standard protocol. Single-tube long fragment read (stLFR) [2] and Hi-C libraries were constructed following the 


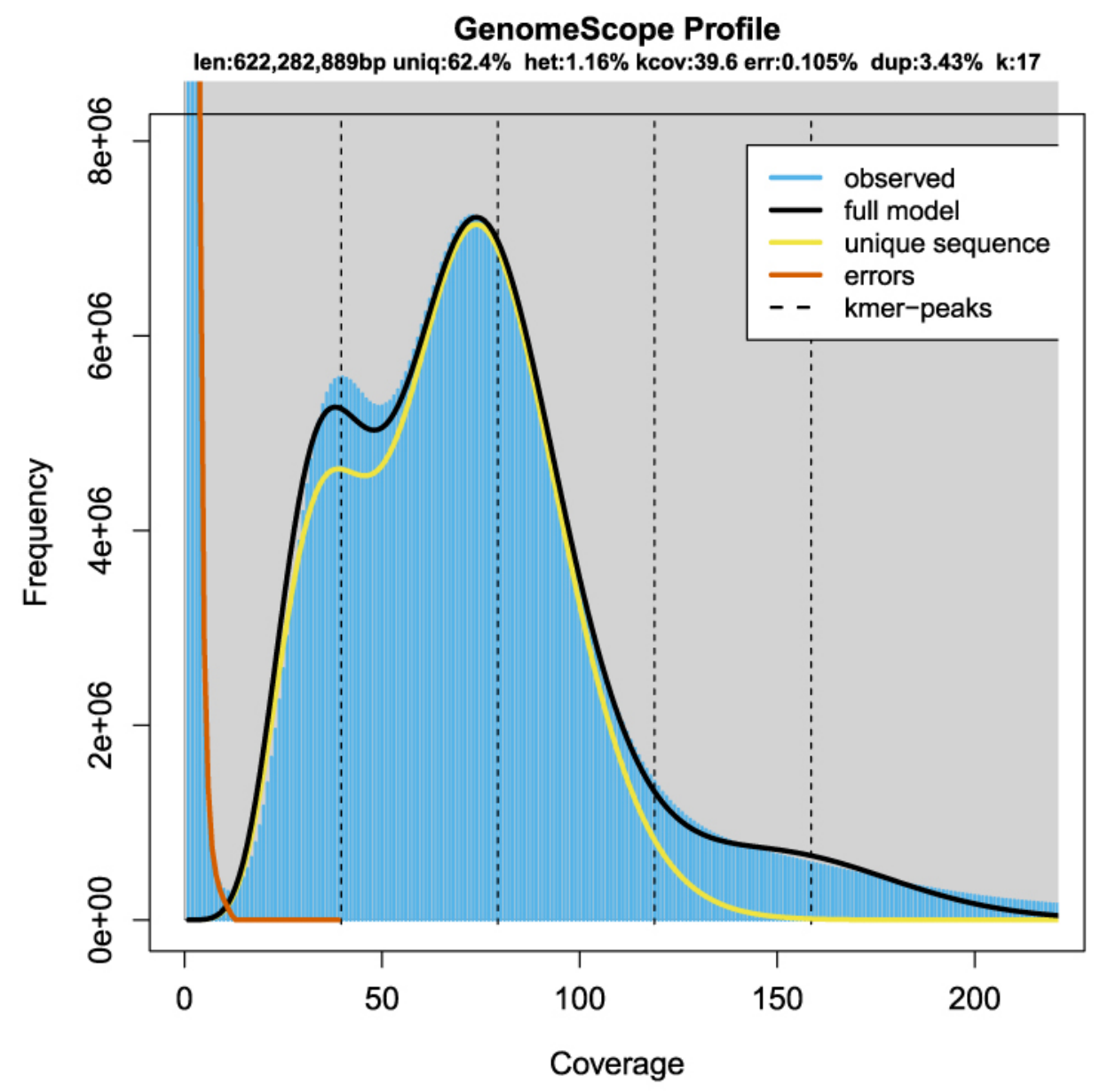

Figure 3. The 17-mer depth distribution of Centropyge bicolor. The estimated genome size is $662.27 \mathrm{Mbp}$ and the heterozygosity is $1.16 \%$.

manufacturers' instructions [2,3] to sequence and assemble the genome. We obtained 130.47 Gbp (gigabase pairs; 197×) raw stLFR data and 134.57 Gbp ( 203.20×) raw Hi-C data (Table 1) using the BGISEQ-500 platform in 100-bp (basepair) paired-end mode.

Low-quality reads (sequences with more than $40 \%$ of bases with a quality score lower than 8), polymerase chain reaction (PCR) duplications, adaptor sequences and reads with a high (greater than 10\%) proportion of ambiguous bases (Ns) occurring in stLFR data were filtered using SOAPnuke (v1.6.5; RRID:SCR_015025) [4]. We obtained $62.6 \mathrm{Gbp}(\sim 91.67 \times)$ clean data (Table 1) to assemble the draft genome. Meanwhile, HiC-Pro (v. 2.8.0) [5] was used for the quality control of raw Hi-C data, and $42.51 \mathrm{Gbp}(\sim 64.19 \times)$ valid data were used to assemble the genome to the chromosome-level (Table 1).

\section{Genome assembly}

Using GenomeScope software (RRID:SCR_017014) with stLFR clean data, $k$-mer distribution was used to understand the genome complexity before genome assembly [6]. The genome size of $C$. bicolor was estimated as $662.27 \mathrm{Mbp}$ (megabase pairs), with $37.6 \%$ repeat sequences and $1.16 \%$ heterozygous sites (Table 2, Figure 3 ). 


\begin{tabular}{|c|c|c|c|c|c|}
\hline \multirow[b]{2}{*}{ Libraries } & \multirow[b]{2}{*}{ Read length } & \multicolumn{2}{|c|}{ Raw data } & \multicolumn{2}{|c|}{ Valid data } \\
\hline & & $\begin{array}{l}\text { Total bases } \\
\text { (Gbp) }\end{array}$ & $\begin{array}{c}\text { Sequencing } \\
\text { depth }(x)\end{array}$ & $\begin{array}{l}\text { Total bases } \\
\text { (Gbp) }\end{array}$ & $\begin{array}{c}\text { Sequencing } \\
\text { depth }(x)\end{array}$ \\
\hline stLFR & 100:100 & 130.47 & 197.00 & 60.71 & 91.67 \\
\hline $\mathrm{Hi}-\mathrm{C}$ & $100: 100$ & 134.57 & 203.20 & 42.51 & 64.19 \\
\hline
\end{tabular}

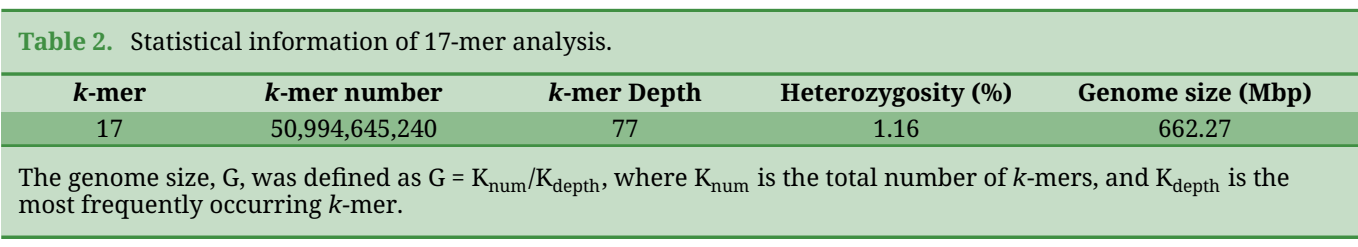

Table 3. Statistics of the draft assembly with stLFR data.

\begin{tabular}{|ccc|}
\hline Statistics & Contig & Scaffold \\
\hline Total number (\#) & 40,442 & 29,065 \\
\hline Total length (bp) & $655,705,062$ & $681,285,455$ \\
Gap (N) (bp) & 0 & $25,580,393$ \\
\hline Average length (bp) & $16,213.47$ & $23,440.06$ \\
\hline N50 length (bp) & 115,524 & $4,424,004$ \\
\hline N90 length (bp) & 6,029 & 7,618 \\
\hline Maximum length (bp) & $1,148,507$ & $21,943,074$ \\
\hline Minimum length (bp) & 48 & 940 \\
\hline GC content (\%) & 41.74 & 41.74 \\
\hline
\end{tabular}

We reformatted the clean stLFR data into $10 \times$ Genomics format using an in-house script [7] and assembled the draft genome using Supernova (v.2.0.1, RRID:SCR_016756) [8] with default parameters. The draft genome was $681 \mathrm{Mbp}$, with a contig N50 of $115.5 \mathrm{Kbp}$ (kilobase pairs) and scaffold N50 of $4.4 \mathrm{Mbp}$ (Table 3), which is similar to the estimated genome size.

To obtain the chromosome-level genome, we used Juicer (v3, RRID:SCR_017226) [9] to build a contact matrix and 3dDNA (v.170123) [10] to sort and anchor scaffolds with the parameters: “-m haploid -s 4-c 24”. There are 24 distinct contact blocks, which correspond to 24 chromosomes, representing $96 \%$ of the whole genome (Figures $4 \mathrm{~A}$, 5, Table 4). On evaluating the completeness of the genome and gene set using Benchmarking Universal Single-Copy Orthologs (BUSCO, v.3.0.2, RRID:SCR_015008) [11] and a vertebrata database, our assembly maintained a score of $96.2 \%$ (Table 5). We also identified putative homologous chromosomal regions between C. bicolor and Oryzias latipes by MCscanx [12] (Figure 6).

In addition, we cut off partial stLFR reads ( $25 \mathrm{M})$ for assembly by MitoZ with default parameters [13] and obtained a 16,961-bp circular mitochondrial genome of $C$. bicolor. Thirteen protein-coding genes, 24 tRNA genes and three rRNA genes were annotated by GeSeq (RRID:SCR_017336) [14] (Figure 4B).

\section{Genomic annotation}

For the annotation of repeats, we carried out homolog annotation and ab initio prediction independently. RepeatMasker (v.4.0.6, RRID:SCR_012954) [15], RepeatProteinMask (a module from RepeatMasker) and trf (Tandem Repeats Finder, v.4.07b) [16] were used to 

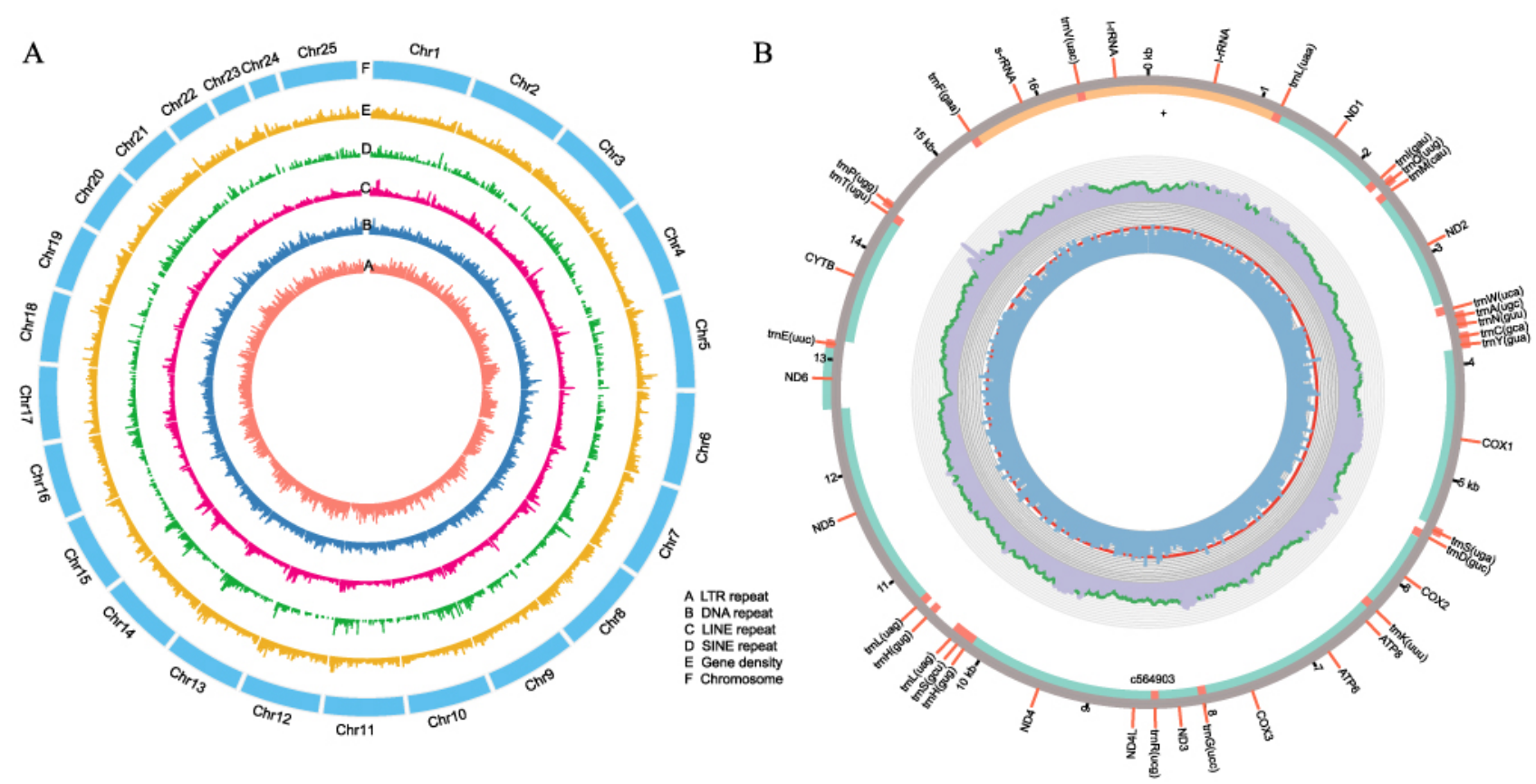

Figure 4. Annotation of the Centropyge bicolor genome. (A) Basic genomic elements of the Centropyge bicolor genome. LTR, long terminal repeat; LINE, long interspersed nuclear elements; SINE, short interspersed elements. (B) Physical map of mitochondrial assembly.

\begin{tabular}{ccc|}
\hline Table 4. Statistics of the chromosome-level genome. & \\
\hline Statistics & Contig & Scaffold \\
Total number (\#) & 40,778 & 28,555 \\
Total length (bp) & $655,705,062$ & $680,873,932$ \\
Gap (N) (bp) & 0 & $25,168,870$ \\
Average length (bp) & $16,079.87$ & $23,844.30$ \\
N50 length (bp) & 113,563 & $21,943,074$ \\
N90 length (bp) & 5,988 & 7,542 \\
Maximum length (bp) & $1,148,507$ & $28,105,280$ \\
Minimum length (bp) & 43 & 43 \\
GC content (\%) & 41.74 & 41.74 \\
\hline
\end{tabular}

identify known repetitive sequences by comparing the whole genome with RepBase [17]. LTR_FINDER (v.1.06, RRID:SCR_015247) [16, 18] and RepeatModeler (v.1.0.8, RRID:SCR_015027) [19] were used in de novo prediction. We also classified transposable elements (TEs) from the integration of all repeats. In total, we identified $124 \mathrm{Mbp}$ (18.32\% of the entire genome) of repetitive sequences (Figure 4A, Table 6), including $110 \mathrm{Mbp}$ of TEs (Figure 4A, Table 7).

Homolog-based and $a b$ initio prediction were used to identify the protein-coding genes. Augustus (v.3.3, RRID:SCR_008417) [20] was used in ab initio prediction basing on a repeat-masked genome [21]. Protein sequences of Astatotilapia calliptera, Danio rerio, Larimichthys crocea, and Oreochromis niloticus were downloaded from the National Center for Biotechnology Information (NCBI) GenBank database and aligned to the $C$. bicolor genome for homolog gene annotation with Genewise (v2.4.1, RRID:SCR_015054) [22]. Finally, we used GLEAN [23] to integrate all the above evidence and obtained a total of 21,774 genes, 


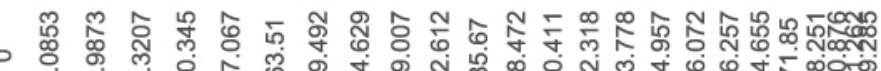

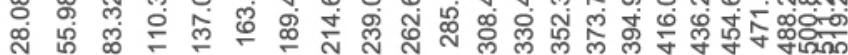

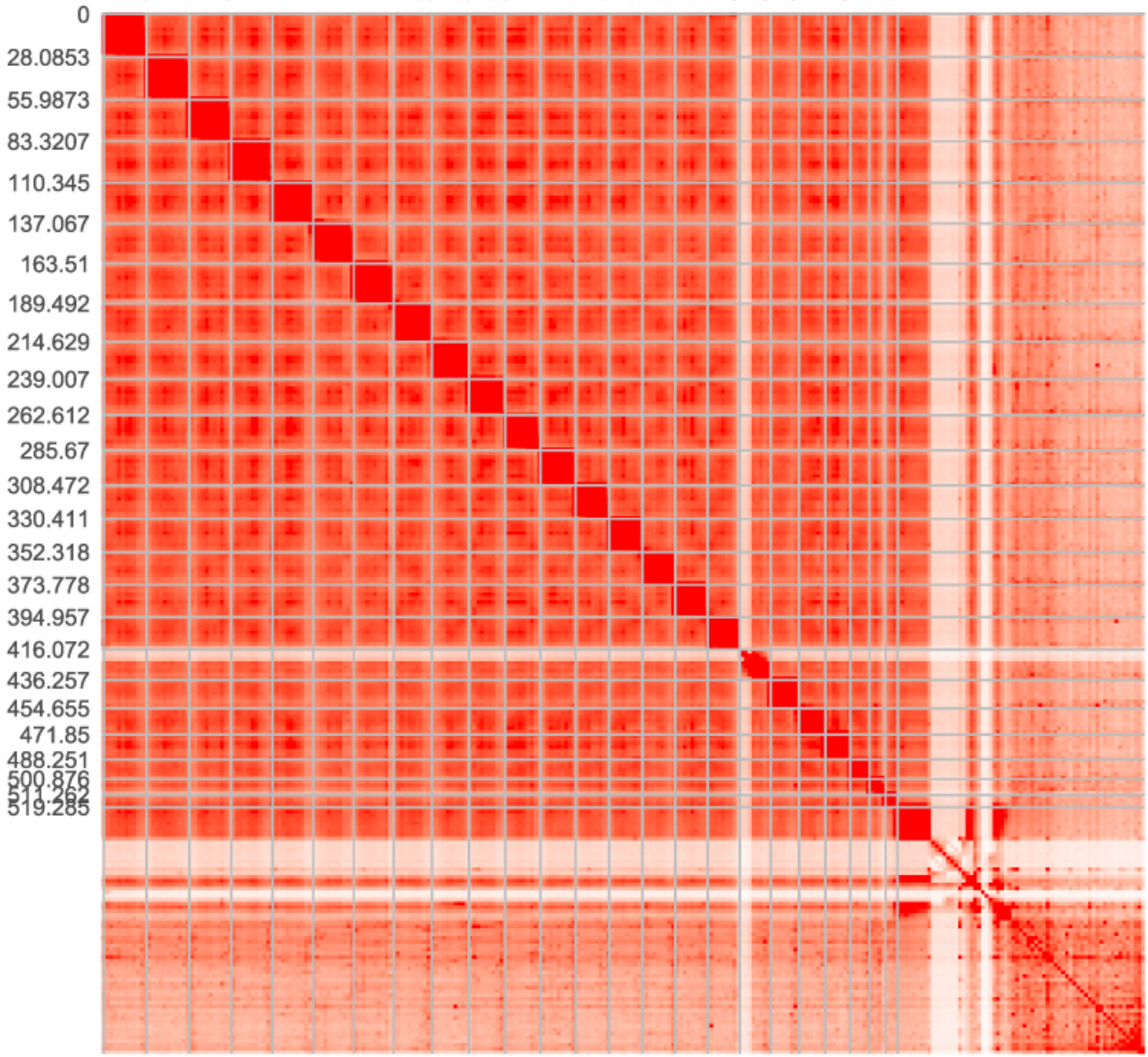

Figure 5. Heat map of interactive intensity between chromosome sequences.

\begin{tabular}{ccccc}
\hline \multicolumn{2}{l}{ Table 5. Statistics of the BUSCO assessment. } & & & \\
\hline & Gene set & & Assembly & \\
Types of BUSCOs & Number & Percentage (\%) & Number & Percentage (\%) \\
Complete BUSCOs & 2,408 & 93.1 & 2,486 & 96.2 \\
Complete single-copy BUSCOs & 2,348 & 90.8 & 2,438 & 94.3 \\
Fragmented BUSCOs & 81 & 3.1 & 64 & 2.5 \\
Missing BUSCOs & 97 & 3.8 & 36 & 1.3 \\
Total BUSCO groups searched & 2,586 & 100 & 2,586 & 100 \\
\hline
\end{tabular}

Table 6. Statistics of repetitive sequences.

\begin{tabular}{|ccc|}
\hline Type & Repeat size (bp) & Percentage of genome (\%) \\
TRF & $14,165,095$ & 2.08 \\
RepeatMasker & $43,423,877$ & 6.38 \\
RepeatProteinMask & $12,503,750$ & 1.84 \\
De novo & $110,871,693$ & 16.28 \\
Total & $124,708,977$ & 18.32 \\
\hline
\end{tabular}




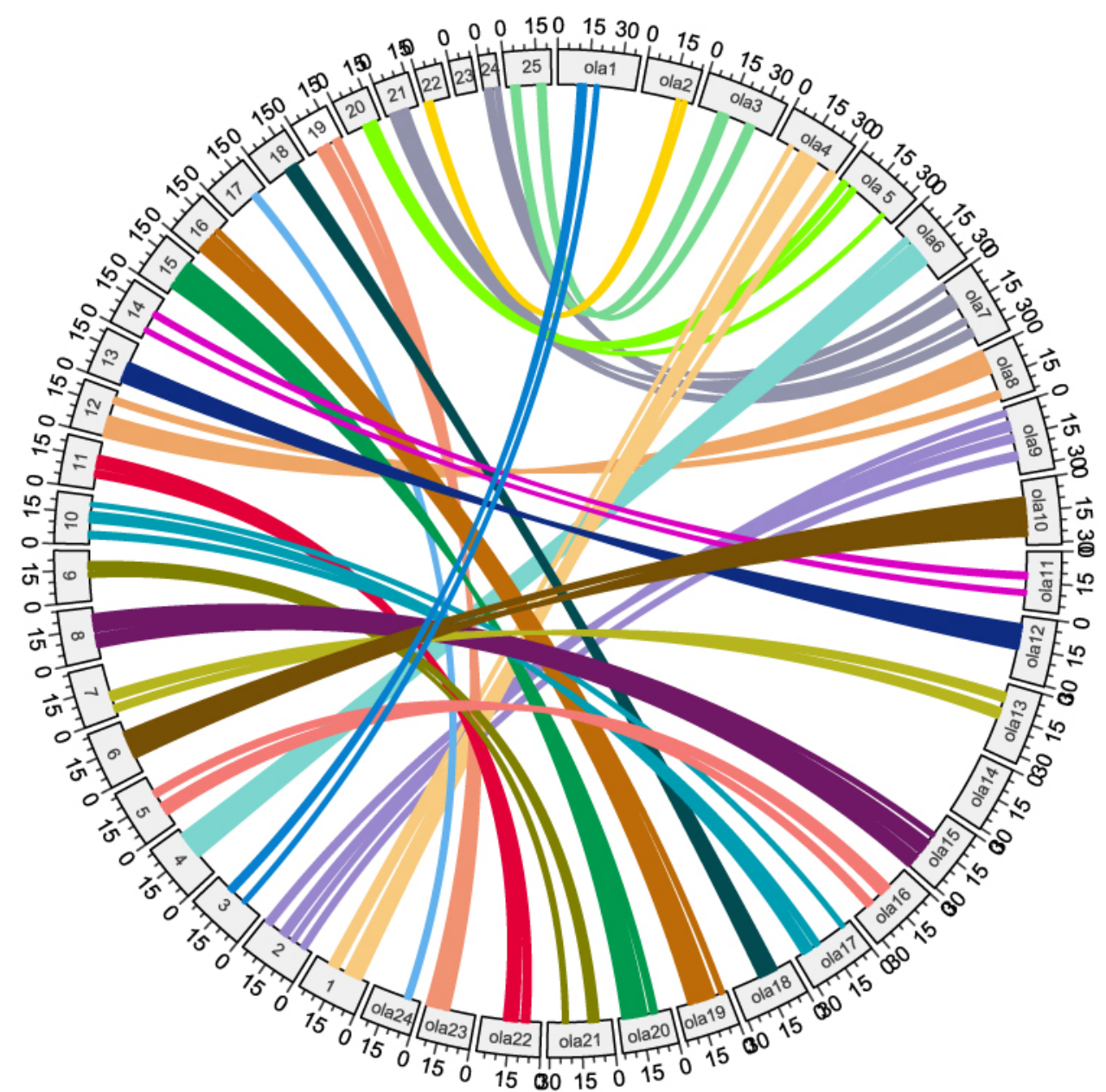

Figure 6. Homologous chromosomal regions between Centropyge bicolor and Oryzias latipes.

\begin{tabular}{ccccc}
\hline \multicolumn{6}{l}{ Table 7. Statistics of transposable elements. } \\
\hline & Repbase TEs, $\boldsymbol{n}(\%)$ & Protein TEs, $\boldsymbol{n}(\%)$ & De novo TEs, $\boldsymbol{n}(\%)$ & Combined TEs, $\boldsymbol{n}(\%)$ \\
DNA & $27,163,851(3.990)$ & $1,068,990(0.157)$ & $61,731,447(9.067)$ & $70,925,963(10.417)$ \\
LINE & $10,228,332(1.502)$ & $6,956,340(1.022)$ & $20,006,579(2.938)$ & $26,714,285(3.924)$ \\
SINE & $856,125(0.126)$ & $0(0.000)$ & $497,024(0.073)$ & $1,187,676(0.174)$ \\
LTR & $10,971,817(1.611)$ & $4,485,808(0.659)$ & $16,270,071(2.390)$ & $23,101,529(3.393)$ \\
Other & $10,041(0.001)$ & 0 & 0 & $10,041(0.001)$ \\
Unknown & 0 & 0 & $14,054,230(2.064)$ & $14,054,230(2.064)$ \\
Total & $43,423,877(6.378)$ & $12,503,750(1.836)$ & $99,265,690(14.579)$ & $109,868,166(16.136)$ \\
\hline
\end{tabular}

which contained 11 exons on average and had an average coding sequence (CDS) length of 1,575 bp (Table 8).

To predict gene functions, 21,774 genes were aligned against several public databases, including TrEMBL [24], SwissProt [24], KEGGViewer [25] and InterProScan [26]. As a result, $99.67 \%$ of all genes were predicted functionally (Table 9, Figure 7). 


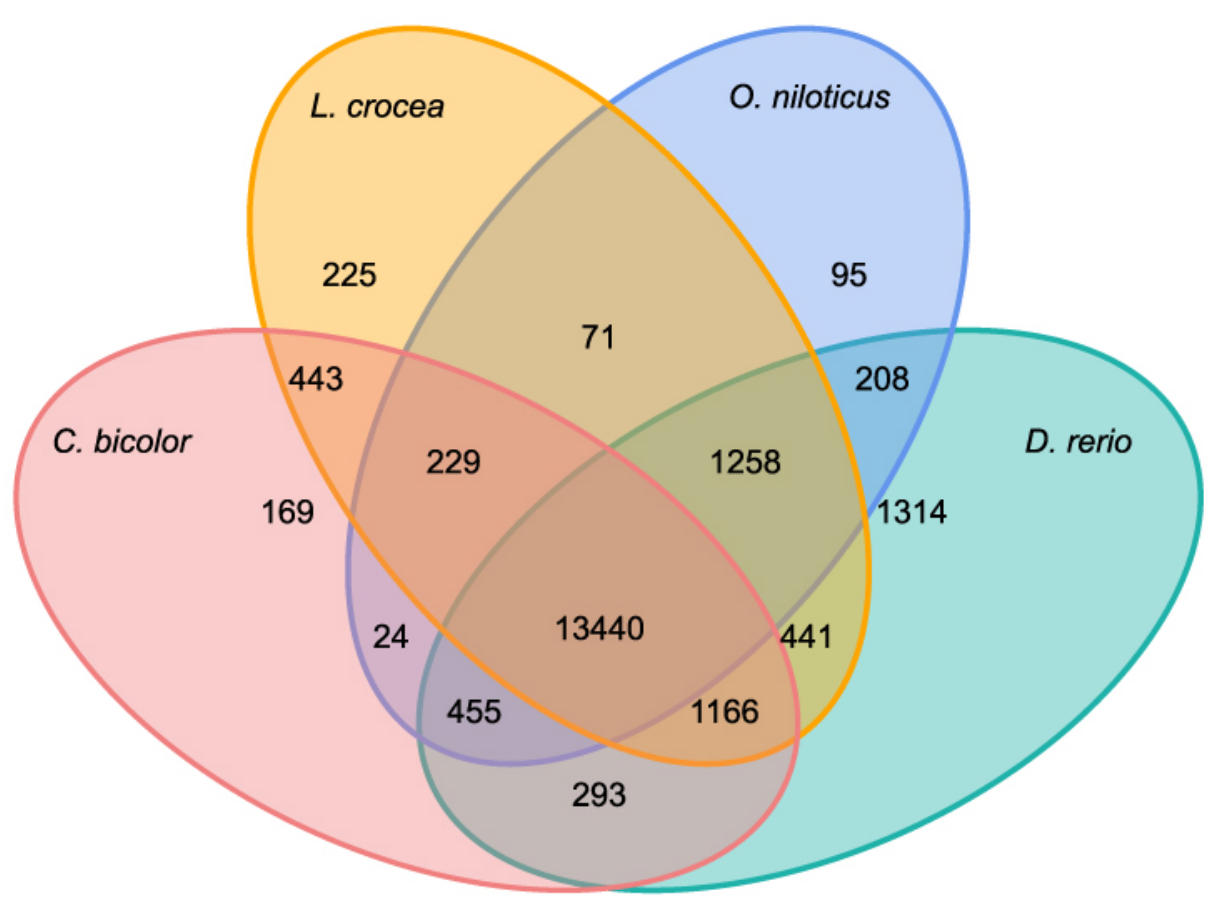

Number of gene families

Figure 7. Venn diagram of orthologous gene families. Four teleost species (Centropyge. bicolor, Larimichthys crocea, Oreochromis niloticus, and Danio rerio) were used to generate the Venn diagram based on gene family cluster analysis.

\begin{tabular}{|c|c|c|c|c|c|c|c|}
\hline & Gene set & Gene number & $\begin{array}{c}\text { Average transcript } \\
\text { length (bp) }\end{array}$ & $\begin{array}{l}\text { Average CDS } \\
\text { length (bp) }\end{array}$ & $\begin{array}{l}\text { Average intron } \\
\text { length (bp) }\end{array}$ & $\begin{array}{l}\text { Average exon } \\
\text { length (bp) }\end{array}$ & $\begin{array}{l}\text { Average exons } \\
\text { per gene }\end{array}$ \\
\hline \multirow[t]{4}{*}{ Homolog } & Astatotilapia calliptera & 51,174 & $21,762.29$ & $2,259.23$ & $1,691.33$ & 180.29 & 12.53 \\
\hline & Danio rerio & 22,005 & $27,982.75$ & $1,570.36$ & $3,438.82$ & 180.90 & 8.68 \\
\hline & Larimichthys crocea & 47,419 & $19,884.78$ & $2,139.39$ & $1,575.94$ & 174.50 & 12.26 \\
\hline & Oreochromis niloticus & 47,067 & $17,771.04$ & $1,906.97$ & $1,608.29$ & 175.53 & 10.86 \\
\hline De novo & Augustus & 34,470 & $9,675.42$ & $1,335.20$ & $1,344.81$ & 185.40 & 7.20 \\
\hline GLEAN & & 21,774 & $14,024.40$ & $1,906.28$ & $1,206.07$ & 172.55 & 11.05 \\
\hline
\end{tabular}

\section{Phylogenetic analysis}

We downloaded the gene data of seven representative teleost fishes from NCBI to study the phylogenetic relationships between $C$. bicolor. These seven fishes were: Danio rerio, Gasterosteus aculeatus, Gadus morhua, Larimichthys crocea, Oryzias latipes, Oreochromis niloticus and Tetraodon nigroviridis. For each dataset, the longest transcripts were selected and aligned to each other by BLASTP (v2.9.0, RRID:SCR_001010) [27] ( $E$-value $\leq 1 \mathrm{e}-5$ ). TreeFam (v.2.0.9, RRID:SCR_013401) [28] was used to cluster gene families, with default parameters. Among all 20,706 clustered gene families, there were 4,450 common single-copy families and 57 families specific to C. bicolor (Table 10). With single-copy sequences, we used PhyML (v.3.3, RRID:SCR_014629) [29] to construct the phylogenetic tree of $C$. bicolor and the seven other fishes mentioned above, setting $D$. rerio as an outgroup. 


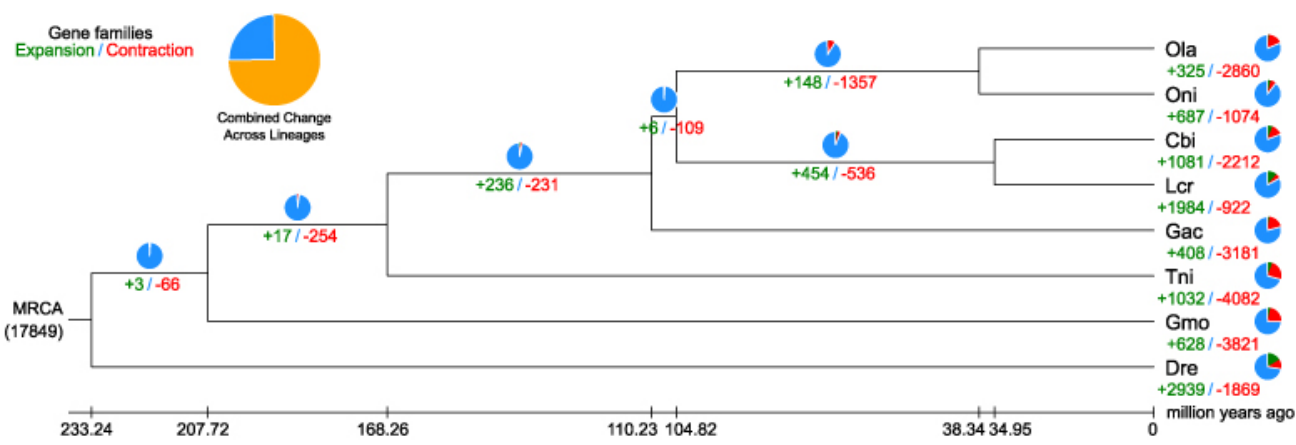

Figure 8. Comparative analysis of the Centropyge bicolor genome. (A) The protein-coding genes of the eight species were clustered into 17,849 gene families. Among these gene families, 4,450 were single-copy gene families. (B) Phylogenetic analysis of Centropyge bicolor (Cbi.), Danio rerio (Dre.), Gasterosteus aculeatus (Gac.), Gadus morhua (Gmo.), Larimichthys crocea (Lcr.), Oryzias latipes (Ola.), Oreochromis niloticus (Oni.), and Tetraodon nigroviridis (Tni.) using single-copy gene families. The species differentiation time between Centropyge bicolor and Larimichthys crocea was $\sim 34.95$ million years.

\begin{tabular}{|ccc|}
\hline Table 9. Statistics of the functional annotation. & & \\
\hline Database & Number & Percentage (\%) \\
\hline Total & 21,774 & 100.00 \\
\hline SwissProt & 20,784 & 95.45 \\
KEGG & 19,168 & 88.03 \\
\hline TrEMBL & 21,688 & 99.61 \\
\hline Interpro & 20,153 & 92.56 \\
\hline Overall & 21,702 & 99.67 \\
\hline
\end{tabular}

\begin{tabular}{|c|c|c|c|c|c|}
\hline Species & $\begin{array}{l}\text { Total } \\
\text { genes }\end{array}$ & $\begin{array}{l}\text { Unclustered } \\
\text { genes }\end{array}$ & Families & $\begin{array}{l}\text { Unique } \\
\text { families }\end{array}$ & $\begin{array}{l}\text { Average number of } \\
\text { genes per family }\end{array}$ \\
\hline Centropyge bicolor & 21,774 & 694 & 16,219 & 57 & 1.3 \\
\hline Danio rerio & 30,067 & 2,188 & 18,575 & 726 & 1.5 \\
\hline Gasterosteus aculeatus & 20,756 & 784 & 15,921 & 16 & 1.25 \\
\hline Gadus morhua & 19,987 & 535 & 15,630 & 9 & 1.24 \\
\hline Larimichthys crocea & 24,403 & 610 & 17,273 & 55 & 1.38 \\
\hline Oryzias latipes & 19,535 & 1,048 & 14,805 & 87 & 1.25 \\
\hline Oreochromis niloticus & 21,431 & 180 & 15,780 & 14 & 1.35 \\
\hline Tetraodon nigroviridis & 19,544 & 901 & 14,803 & 57 & 1.26 \\
\hline
\end{tabular}

Based on the phylogenetic tree and single-copy sequences, the divergence time between different species was estimated by MCMCTREE with parameters of "-model 0 -rootage 500 -clock 3". The results showed that $C$. bicolor was formed $\sim 34.95$ million years ago, when differentiated from the common ancestor with L. crocea (Figure 8).

\section{Analysis of bicolor formation in teleosts}

Current studies suggest that different pigment cells produce different pigments. Some types of pigment cells already have been identified in teleost [30]. C. bicolor has an attractive body color with clear color boundaries, but the molecular mechanism underlying this remains unknown. Compared with other teleost, there are 1,081 expanded gene families and 57 specific gene families in C. bicolor (Figure 9). Functional enrichment analysis 


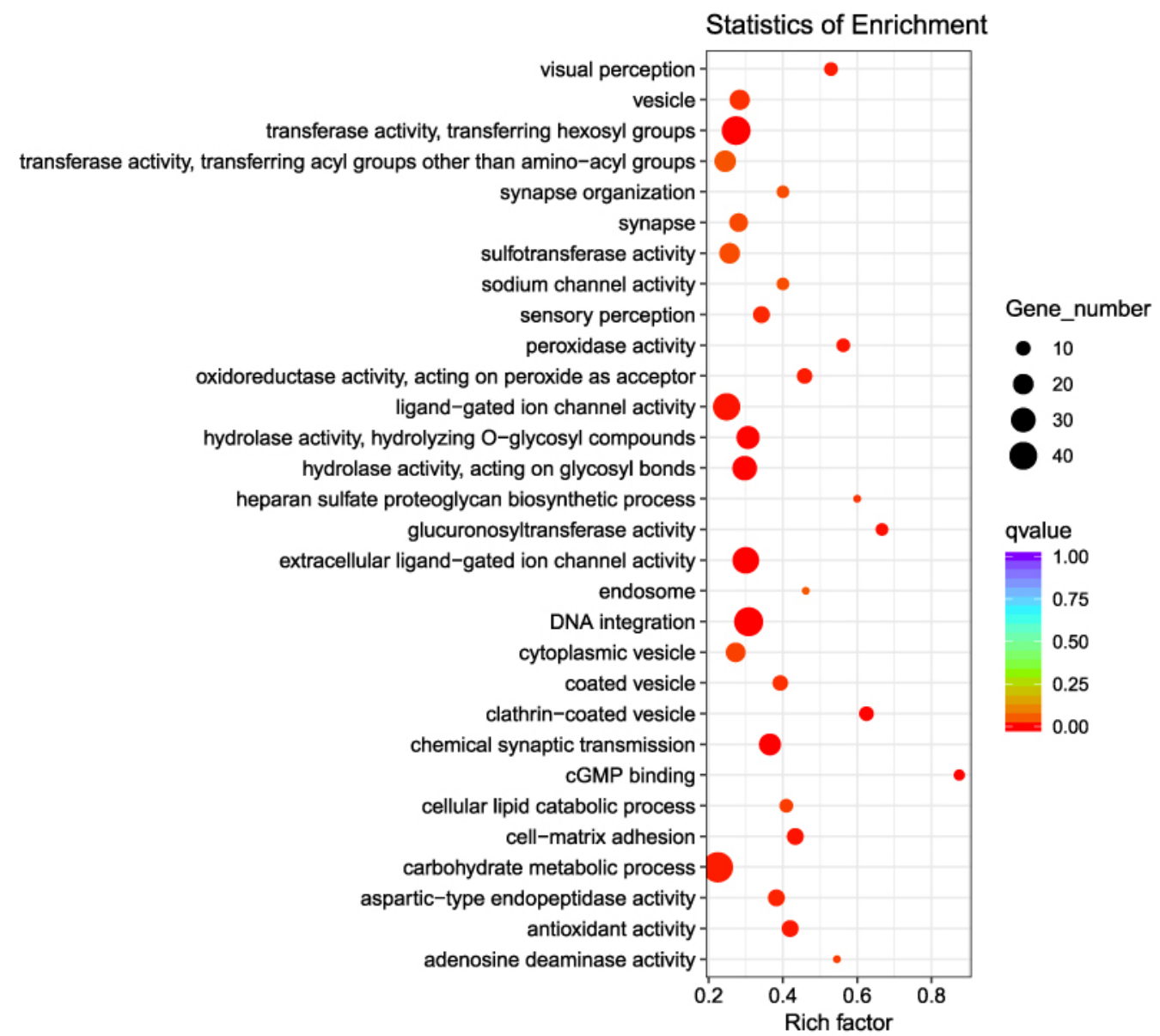

Figure 9. Statistics of gene function enrichment (Gene Ontology) for expanded genes of Centropyge bicolor. Nodes are colored by $q$-value (adjusted $p$-value). Node size is shown according to its enriched gene number.

showed that notable expansion occurred in those gene families related to visual development and enzyme metabolism (Figure 9).

\section{RE-USE POTENTIAL}

Coral reef fishes, with distinctive color patterns and color morphs, are important for understanding the adaptive evolution of fishes. In this study, we firstly assembled a high-quality, chromosome-level genome of $C$. bicolor, with a length of $681 \mathrm{Mbp}$, and annotated 21,774 genes. This is the first genome of a fish from the Pomacanthidae family. These genomic data will be useful for genome-scale comparisons and further studies on the mechanisms underlying colorful body development and adaptation.

\section{DATA AVAILABILITY}

The data sets supporting the results of this article are available in the GigaScience Database [31]. Raw reads from genome sequencing and assembly are deposited at the China National Gene Bank under reference number CNP0001160, which contains sample 
information (CNS0315939), Hi-C raw data (CNX0286336) and stLFR raw data (CNX0286337). The project also has been deposited at NCBI under accession ID PRJNA702283.

\section{DECLARATIONS}

\section{LIST OF ABBREVIATIONS}

bp: base pair; BUSCO: Benchmarking Universal Single-Copy Orthologs; Gbp: gigabase pair; Kbp: kilobase pair; KEGG: Kyoto Enyclopedia of Genes and Genomes; Mbp: megabase pair; NCBI: National Center for Biotechnology Information; stLFR: single-tube long fragment reads; TE: transposable element.

\section{ETHICAL APPROVAL}

All resources used in this study were approved by the Institutional Review Board of BGI (IRB approval No. FT17007). This experiment has passed the ethics audit of the Beijing Genomics Institute (BGI) Gene Bioethics and Biosecurity Review Committee.

\section{CONSENT FOR PUBLICATION}

Not applicable.

\section{COMPETING INTERESTS}

The authors declare that they have no competing interests.

\section{FUNDING}

This work was supported by funding from the "Blue Granary" project for scientific and technological innovation of China (2018YFD0900301-05).

\section{AUTHORS' CONTRIBUTIONS}

H.Z. and G.F. designed this project. M.Z. prepared the samples. S.L., S.P., W.X., C.W. and C.M. conducted the experiments. C.L., X.Y., L.S., R.Z. and Q.L. did the analyses. C.L., X.Y., L.S., R.Z. wrote and revised the manuscript. All authors read and approved the final version of the manuscript.

\section{ACKNOWLEDGEMENTS}

We thank the China National Genebank for technical support in constructing and sequencing the stLFR library.

\section{REFERENCES}

1 Mendoncą RC, Chen JY, Zeng C, Tsuzuki MY, Embryonic and early larval development of two marine angelfish, Centropyge bicolor and Centropyge bispinosa. Zygote, 2020; 28(3): 196-202. doi:10.1017/S0967199419000789.

2 Li C et al. Protocols for "Bicolor Angelfish (Centropyge bicolor) genome provided first chromosome-level reference of Pomacanthidae family and clues for bi-color body formation”. protocols.io. 2020; https://dx.doi.org/10.17504/protocols.io.bpxhmpj6.

3 Wang $\mathrm{O}$ et al. Efficient and unique cobarcoding of second-generation sequencing reads from long DNA molecules enabling cost-effective and accurate sequencing, haplotyping, and de novo assembly. Genome Res., 2019; 29(5): 798-808. doi:10.1101/gr.245126.118.

4 Chen Y et al. SOAPnuke: A MapReduce acceleration-supported software for integrated quality control and preprocessing of high-throughput sequencing data. Gigascience, 2018; 7(1): gix120. doi:10.1093/gigascience/gix120. 
5 Chen C-J et al. HiC-Pro: an optimized and flexible pipeline for Hi-C data processing. Genome Biol., 2015; 16: 259. doi:10.1186/s13059-015-0831-x.

6 Vurture GW et al. GenomeScope: Fast reference-free genome profiling from short reads. Bioinformatics, 2017; 33(14): 2202-2204. doi:10.1093/bioinformatics/btx153.

7 BGI-QingDao. stlfr2supernova_pipeline. 2021; https://github.com/BGI-Qingdao/stlfr2supernova_pipeline.

8 Wong KHY, Levy-Sakin M, Kwok PY, De novo human genome assemblies reveal spectrum of alternative haplotypes in diverse populations. Nat. Commun., 2018; 9: 3040. doi:10.1038/s41467-018-05513-w.

9 Durand NC et al. Juicer provides a one-click system for analyzing loop-resolution Hi-C experiments. Cell Syst., 2016; 3(1): 95-98. doi:10.1016/j.cels.2016.07.002.

10 Dudchenko $O$ et al. De novo assembly of the Aedes aegypti genome using Hi-C yields chromosome-length scaffolds. Science, 2017; 356(6333): 92-95. doi:10.1126/science.aal3327.

11 Waterhouse RM, Seppey M, Sim FA, Ioannidis P, BUSCO applications from quality assessments to gene prediction and phylogenomics. Letter Fast Track, 2017; doi:10.1093/molbev/msx319.

12 Wang Y et al. MCScanX: a toolkit for detection and evolutionary analysis of gene synteny and collinearity. Nucleic Acids Res., 2012; 40(7): e49. doi:10.1093/nar/gkr1293.

13 Meng G, Li Y, Yang C, Liu S, MitoZ: A toolkit for animal mitochondrial genome assembly, annotation and visualization. Nucleic Acids Res., 2019; 47(11): e63. doi:10.1093/nar/gkz173.

14 Tillich M et al. GeSeq - versatile and accurate annotation of organelle genomes. Nucleic Acids Res., 2017; 45(W1): W6-W11. doi:10.1093/nar/gkx391.

15 Tarailo-Graovac M, Chen N, Using RepeatMasker to identify repetitive elements in genomic sequences. Curr. Protoc. Bioinformatics, 2009; doi:10.1002/0471250953.bi0410s25.

16 Carrillo-Avila M, Resende EK, Marques DKS, Galetti PM, Tandem repeats finder: a program to analyze DNA sequences. Conserv. Genet., 2009; 25: 4.10.1-4.10.14. doi:10.1590/S1679-62252007000200018.

17 Bao W, Kojima KK, Kohany O, Repbase Update, a database of repetitive elements in eukaryotic genomes. Mob. DNA, 2015; 6: 11. doi:10.1186/s13100-015-0041-9.

$18 \mathrm{Xu}$ Z, Wang H, LTR_FINDER: an efficient tool for the prediction of full-length LTR retrotransposons. Nucleic Acids Res., 2007; 35(2): W265-W268. doi:10.1093/nar/gkm286.

19 Storer J, Hubley R, Rosen J, Wheeler TJ, Smit AF, The Dfam community resource of transposable element families, sequence models, and genome annotations. Mob. DNA, 2021; 12: 2. doi:10.1186/s13100-020-00230-y.

20 Stanke M, Schöffmann O, Morgenstern B, Waack S, Gene prediction in eukaryotes with a generalized hidden Markov model that uses hints from external sources. BMC Bioinform., 2006; 7: 62. doi:10.1186/1471-2105-7-62.

21 Stanke M, Keller O, Gunduz I, Hayes A, Waack S, Morgenstern B, AUGUSTUS: ab initio prediction of alternative transcripts. Nucleic Acids Res., 2006; 34(2): W435-W439. doi:10.1093/nar/gkl200.

22 Doerks T, Copley RR, Schultz J, Ponting CP, Bork P, Systematic identification of novel protein domain families associated with nuclear functions. Genome Res., 2002; 12(1): 47-56. doi:10.1101/gr.203201.

23 Lewis S et al. Creating a honey bee consensus gene set. Genome Biol., 2002; 3: research0082.1. doi:10.1186/gb-2002-3-12-research0082.

24 Bairoch A, The SWISS-PROT protein sequence database and its supplement TrEMBL in 2000. Nucleic Acids Res., 2000; 28(1): 45-48. doi:10.1093/nar/28.1.45.

25 Habermann BH, Villaveces JM, Jimenez RC, KEGGViewer, a BioJS component to visualize KEGG pathways. F1000Research, 2014; 3: 43, doi:10.12688/f1000research.3-43.v1.

26 Jones P et al. InterProScan 5: Genome-scale protein function classification. Bioinformatics, 2014; 30(9): 1236-1240. doi:10.1093/bioinformatics/btu031.

27 Altschul SF, Gish W, Miller W, Myers EW, Lipman DJ, Basic local alignment search tool. J. Mol. Biol., 1990; 215(3): 403-410. doi:10.1016/S0022-2836(0580360-2.

28 Ruan J et al. TreeFam: 2008 update. Nucleic Acids Res., 2007; 36(suppl 1): D735-D740. doi:10.1093/nar/gkm1005. 
29 Guindon S, Dufayard JF, Lefort V, Anisimova M, Hordijk W, Gascuel O, New algorithms and methods to estimate maximum-likelihood phylogenies: Assessing the performance of PhyML 3.0. Syst. Biol., 2010; 59(3): 307-321. doi:10.1093/sysbio/syq010.

30 Kimura $\mathbf{T}$ et al. Leucophores are similar to xanthophores in their specification and differentiation processes in medaka. Proc. Natl Acad. Sci. USA, 2014; 111(20): 7343-7348. doi:10.1073/pnas.1311254111.

$31 \mathrm{Li} \mathrm{C}$ et al. Genome data of the bicolor angelfish (Centropyge bicolor). GigaScience Database. 2020; http://dx.doi.org/10.5524/100802. 